\title{
Long-acting oral phosphodiesterase inhibition preconditions against reperfusion injury in an experimental lung transplantation model
}

\author{
Eric S. Weiss, MD, ${ }^{a}$ Hunter C. Champion, MD, PhD, ${ }^{\mathrm{b}}$ Jason A. Williams, MD, ${ }^{\mathrm{a}}$ William A. Baumgartner, MD, ${ }^{\mathrm{a}}$ and \\ Ashish S. Shah, MD
}

\begin{abstract}
Objectives: Ischemia-reperfusion injury remains a devastating complication of lung transplantation. Phosphodiesterase inhibitors have been shown to precondition tissues against ischemia-reperfusion injury. Little is known, however, about the utility of phosphodiesterase inhibition in reperfusion injury after lung transplantation. We evaluated the long-acting phosphodiesterase-5 inhibitor, tadalafil, in an ex vivo lung transplant model.
\end{abstract}

\begin{abstract}
Methods: New Zealand White rabbits $(4 \mathrm{~kg})$, were given oral tadalafil $(\mathrm{n}=11) 24$ hours before lung harvest and compared with rabbits given oral vehicle alone $(\mathrm{n}=11)$. Lungs were recovered with Perfadex solution (Vitrolife, Kungsbacka, Sweden) and cold stored for 18 hours. After storage, lung blocks were reperfused with donor rabbit blood in an ex vivo apparatus. Pulmonary artery pressures were recorded with serial arterial and venous blood gas sampling and animals served as their own controls. Phosphodiesterase-5 and protein kinase G tissue activity assays confirmed drug effects. Luminol chemiluminescence assay was used to measure reactive oxygen species and levels of endothelial and inducible nitric oxide synthase were measured.
\end{abstract}

Results: Extended cold storage, followed by reperfusion produced a consistent reproducible decrease in oxygenation and increase in pulmonary pressure. Tadalafil-treated animals exhibited greater $\mathrm{PaO}_{2}$ throughout the course of reperfusion $(P=.001)$ Mean pulmonary artery pressure was lower in tadalafil-treated animals $(22 \mathrm{vs} 40 \mathrm{~mm}$ $\mathrm{Hg} ; P=.04)$. Phosphodiesterase-5 activity was decreased $(143 \pm 8$ vs $205 \pm 32 \mathrm{mP} ; P<.001)$ with protein kinase $\mathrm{G}$ activity increased $(25 \pm 12 \mathrm{vs} 12 \pm 2.4 \mathrm{fU} / \mu \mathrm{g} ; P=.01)$ in the experimental group confirming that oral pretreatment resulted in active phosphodiesterase inhibition in the lung tissue. Reactive oxygen species (as measured by luminol activity) were decreased in tadalafil-treated animals ( $7.8 \pm 1.5$ vs $10.2 \pm 1.2$ relative light units; $P=.003)$.

Conclusions: Our experimental model demonstrates that oral donor pretreatment with a long-acting phosphodiesterase inhibitor is an effective strategy for improving pulmonary performance after reperfusion. Importantly, phosphodiesterase enzymes and their downstream effectors may play a critical role in reperfusion injury after lung transplantation.

Despite 20 years of successful lung transplantation, primary graft dysfunction (PGD) remains a significant and devastating cause of early postoperative respiratory failure. ${ }^{1,2}$ Occurring in up to $20 \%$ of lung transplant recipients, PGD is

From the Division of Cardiac Surgery, ${ }^{\text {a }}$ Department of Surgery, and the Division of Cardiology, ${ }^{b}$ Department of Medicine, The Johns Hopkins Medical Institutions, Baltimore, Md.

Supported by the Mildred and Carmont Blitz Cardiac Research Fund, the Joyce Koons Family Cardiac Endowment Fund, the American Medical Association Foundation (AMA seed grant, E.S.W.), and the National Institutes of Health (NIH 2T32DK007713-12 ESW). Eric Weiss and Jason Williams are Irene Piccinini Investigators in Cardiac Surgery. The study was also supported in part by an American Heart Association Scientist Development Grant, a grant from the W.W.Smith Charitable Trust, a Shih-Chun Wang Young Investigator Award; a Giles F. Filley Award of the American Physiological Society; the Bernard Family Foundation, and NIHP50HL084946 (H.C.C.).

Read at the Eighty-eighth Annual Meeting of The American Association for Thoracic Surgery, San Diego, Calif, May 10-14, 2008.

Received for publication May 13, 2008; revisions received Nov 25, 2008; accepted for publication Dec 30, 2008.

Address for reprints: Ashish S. Shah, MD, Assistant Professor of Surgery, Division of Cardiac Surgery, The Johns Hopkins Hospital, Blalock 618, 600 N. Wolfe St, Baltimore, MD 21287 (E-mail: ashah29@jhu.edu).

J Thorac Cardiovasc Surg 2009;137:1249-57

$0022-5223 / \$ 36.00$

Copyright (C) 2009 by The American Association for Thoracic Surgery

doi:10.1016/j.jtcvs.2008.12.040 characterized by the development of progressive hypoxemia, increased pulmonary pressures, and pulmonary edema. ${ }^{3}$ Patients in whom PGD develops have increased rates of rejection and infection, longer intensive care unit and hospital lengths of stay, and greater short- and longterm mortality. ${ }^{1,2,4-7}$

Alterations in pulmonary vascular resistance, microvascular permeability, and gas exchange support the belief that injury in PGD is primarily caused by ischemia and reperfusion (IR). ${ }^{8}$ Several studies have suggested an important role for nitric oxide (NO) signaling pathways mediated by secondary messengers such as cyclic guanosine monophosphate (GMP). Models of lung IR injury have demonstrated that reduced levels of cyclic GMP ${ }^{9}$ and low levels of cyclic GMP after lung reperfusion have been associated with the development of pulmonary hypertension, ${ }^{10}$ reduced oxygenation, ${ }^{11}$ and increased microvascular permeability. ${ }^{8,10}$

The importance of NO signaling via cyclic GMP suggests an opportunity for preconditioning via modulation of this pathway. The phosphodiesterase (PDE) enzymes represent a class of 11 distinct isoenzymes responsible for hydrolysis of cyclic nucleotides. ${ }^{12}$ Of these, PDE5 is perhaps the best known because of its high specificity for cyclic GMP and 


\begin{tabular}{|c|c|}
\hline \multicolumn{2}{|c|}{ Abbreviations and Acronyms } \\
\hline eNOS & $=$ endothelial nitric oxide synthase \\
\hline GEE & $=$ generalized estimating equation \\
\hline GMP & $=$ guanosine monophosphate \\
\hline iNOS & $=$ inducible nitric oxide synthase \\
\hline IR & $=$ ischemia and reperfusion \\
\hline NO & $=$ nitric oxide \\
\hline NOS & $=$ nitric oxide synthase \\
\hline PA & $=$ pulmonary artery \\
\hline PAP & $=$ pulmonary artery pressure \\
\hline $\mathrm{PCO}_{2}$ & $=$ partial pressure of carbon dioxide \\
\hline PDE & $=$ phosphodiesterase \\
\hline PGD & $=$ primary graft dysfunction \\
\hline PKG & $=$ protein kinase $\mathrm{G}$ \\
\hline $\mathrm{PO}_{2}$ & $=$ partial pressure of oxygen \\
\hline RM-AI & $\begin{aligned}= & \text { repeated-measures analysis of } \\
& \text { variance }\end{aligned}$ \\
\hline ROS & $=$ reactive oxygen species \\
\hline
\end{tabular}

availability of specific Food and Drug Administration-approved inhibitors used primarily for the treatment of erectile dysfunction and pulmonary hypertension. Through augmentation of cyclic GMP levels, PDE inhibition may protect against the deleterious effects of IR injury after lung transplantation.

Tadalafil (Cialis; Lilly ICOS, Indianapolis, Ind) is a selective PDE5 inhibitor that was approved for the treatment of erectile dysfunction in 2003. Tadalafil is distinct from other commonly used selective PDE5 inhibitors (ie, sildenafil or vardenafil) in that it possesses a substantially longer half-life (17.5 hours). ${ }^{13-15}$ Tadalafil is furthermore readily bioavailable when administered orally, reaching peak levels approximately 2 hours after administration. Because of these pharmacokinetic properties, tadalafil is a potentially attractive agent for donor preconditioning before lung transplantation.

Although much research has focused on treatment of IR at the recipient level, few studies have investigated donor preconditioning as a means to prevent IR injury after transplantation. Further, to our knowledge, no study has investigated the use of oral tadalafil as a preconditioning agent. Given the potential therapeutic role for PDE inhibition in PGD, we hypothesized that pretreatment with a long-acting oral selective PDE5 inhibitor will reduce reperfusion injury and graft dysfunction in an ex vivo experimental model of lung transplantation.

\section{METHODS \\ Model}

We conducted these experiments with an ex vivo lung reperfusion model whereby rabbit lungs are externally ventilated and perfused with donor rabbit blood. The experiment used male New Zealand White rabbits (Myrtle's
Rabbitry Inc, Thompson Station, Tenn) weighing 3 to $4 \mathrm{~kg}$. All studies were approved by the Animal Care and Use Committee at the Johns Hopkins University.

\section{Experimental Protocol}

Protocol in brief. A total of 26 rabbits were arbitrarily divided into 2 experimental groups and underwent en bloc heart-lung harvest followed by 18 hours of cold storage. These heart-lung blocks were reperfused (18 hours after harvest) with donor rabbit blood for 180 minutes while physiologic data were recorded.

\section{Experimental Groups}

Thirteen animals (experimental group) received $1 \mathrm{mg}$ of oral tadalafil (Toronto Research Chemicals, Inc, North York, Ontario, Canada) dissolved in $0.5 \mathrm{~mL}$ of dimethyl sulfoxide (JHU Core Store; Sigma Chemical Co, St Louis, Mo) 6 hours before lung harvest ( 24 hours before reperfusion). The second set of 13 rabbits (control group) received $1 \mathrm{~mL}$ of oral dimethyl sulfoxide only administered in an identical time frame. Four animals were excluded owing to technical complications relating to surgery. In all 4 cases, a pulmonary artery (PA) injury occurred during harvest (see procedure below) and when it became clear that PA perfusion was compromised, the experiment was aborted with no additional data collected. The final study population thus comprised 22 subjects (11 experimental and 11 controls).

\section{Heart-Lung Block Harvest}

Experimental rabbits were anesthetized with intramuscular injection of ketamine $(35 \mathrm{mg} / \mathrm{kg})$ and xylazine $(6.5 \mathrm{mg} / \mathrm{kg})$ with intravenous acepromazine $(5 \mathrm{mg} / \mathrm{kg})$ given as needed for additional sedation. A tracheotomy was performed for endotracheal intubation and mechanical ventilation (forced expiratory volume in 1 second $100 \%$ ) was initiated (Harvard ventilator apparatus, model 665; Harvard Apparatus Co, Holliston, Mass). Through a midline incision and medium sternotomy, the chest was entered and the superior and inferior venae cavae, aorta, and PA were isolated. Intravenous heparin $(1000 \mathrm{U} / \mathrm{kg})$ was given, and $30 \mu \mathrm{g}$ of prostaglandin $\mathrm{E}_{1}$ was administered directly into the PA. The PA was cannulated via a right ventriculotomy. An incision was made within the left ventricle for venting, and cold $\left(4^{\circ} \mathrm{C}\right)$ Perfadex solution (Vitrolife, Kungsbacka, Sweden) was run by gravity drainage into the lungs through the PA cannula. The superior and inferior venae cavae and aorta were ligated and a left atrial cannula was placed through the left ventricle. After placement of cannulas, cold ice slush was placed surrounding the heart-lung block, and this block was excised from within the mediastinum. The lungs were inflated, and the block was placed within a bag of Perfadex solution and cold stored $\left(4^{\circ} \mathrm{C}\right)$ for 18 hours.

\section{Perfusion Pump System}

After cold storage, the heart-lung block was suspended by the trachea and ventilated at $10 \mathrm{~mL} / \mathrm{kg}, 30$ breaths $/ \mathrm{min}$, at $100 \%$ forced expiratory volume in 1 second. Two donor rabbits were heparinized $(1000 \mathrm{U} / \mathrm{kg})$ and exsanguinated to obtain approximately $300 \mathrm{~mL}$ of whole blood. All animals were perfused for 180 minutes with this donor blood using a Sarns 5000 roller head pump (Sarns, Inc, Ann Arbor, Mich). Arterial blood removed from the left atrial cannula was collected in a reservoir and deoxygenated to achieve a $\mathrm{PO}_{2}$ and $\mathrm{PCO}_{2}$ of $60 \mathrm{~mm} \mathrm{Hg}$ (simulating venous blood). Venous blood was then pumped into the PA cannula for reperfusion.

\section{Hemodynamic Measurements}

Physiologic measurements were recorded initially and then every 15 minutes for the full 180 minutes of perfusion. Pressure transducers recorded pressures for fluid-filled lines including PA and left atrial pressures. Arterial and venous blood sampes were collected every 15 minutes for blood gas sampling (arterial blood gas analyzer, model 348, Chiron Diagnostics, Norwood, Mass). 


\section{Lung Samples}

Lung samples were taken at four time points during the experiment: immediately after harvest (before cold storage), immediately before perfusion (after cold storage), and then at 1 and 3 hours after perfusion. Lung samples were removed sharply with the aid of hemoclips for hemostasis (large ligating clips; Weck, Research Triangle Park, NC). Samples were snap frozen in liquid nitrogen and stored at $-80^{\circ} \mathrm{C}$ for later biochemical analysis.

\section{Wet/Dry Ratios}

Wet/dry ratios were obtained to determine levels of pulmonary edema. Right lower lobe samples were removed and weighed after the full 180 minutes of perfusion. These samples were allowed to dry completely and subsequently were reweighed for ratio determination.

\section{PDE5A and Protein Kinase G (PKG) Activity}

Total low-kinetic rate constant $(\mathrm{Km})$ cyclic GMP PDE activity was assayed with a fluorescence polarization assay (Molecular Devices, Sunnyvale, Calif) and results were read on a Spectramax M5 plate reader (Molecular Devices). PKG-1 activity was assayed from lung tissue lysates with a colorimetric analysis (CycLex Co, Ltd, Nagano, Japan). ${ }^{16}$

\section{Cyclic GMP Levels}

Cyclic GMP concentration in whole lung lysates was determined with a commercially available colormetric assay performed according to the manufacturer's specifications (Amersham Pharmaceuticals, Buckinghamshire, United Kingdom). ${ }^{17}$

\section{Reactive Oxygen Species (ROS)}

ROS were examined by use of a luminol enhanced chemiluminescence assay (EMD Biosciences, San Diego, Calif). Fresh frozen lung tissue was homogenized in iced phosphate-buffered saline buffer, centrifuged, and resuspended in assay buffer to a concentration of $100 \mu \mathrm{L}$ luminol. Results were read on a Spectramax M5 plate reader. $^{18}$

\section{Nitric Oxide Synthase (NOS) Activity}

NOS activity was obtained from lung tissue lysates via a [3H]-L-arginine to $[3 \mathrm{H}]-\mathrm{L}$ citrulline conversion (Sigma-Aldrich, St Louis, Mo). ${ }^{17,19}$

\section{Statistical Analysis}

For physiologic data (ie, partial pressure of oxygen, $\mathrm{PO}_{2}$; partial pressure of carbon dioxide, $\mathrm{PCO}_{2}$; and mean pulmonary artery pressure, $\mathrm{PAP}$ ), two independent statistical tests were conducted to assess differences between treatment groups accounting for the longitudinal nature of the repeated measures over time. First, repeated-measures analysis of variance (RM-ANOVA) was performed to examine the effects of tadalafil measured across 13 distinct time points. Post hoc comparisons at specific time points were conducted with the Tukey honest significant difference test. Multilevel random effects modeling was also performed to account for interactions both between and within each animal. The model used (generalized estimating equation, GEE) estimated the variance in the physiologic parameter of interest between animals and across time. Values for both the RM-ANOVA and GEE are presented when discussing treatment effects on physiologic parameters. Results of biochemical activity assays (performed on a subset of animals $(n=15)$ were compared for each point of measurement using the Student $t$ test (due to a priori expectations of differences for each sample). When prestorage and postperfusion values within groups were compared, the paired comparisons Student $t$ test was used. Data are presented as means \pm standard deviation. All statistical analyses were conducted with the aid of STATA software (version 9.2 special edition, Stata Corporation LP, College Station, Tex).

\section{RESULTS \\ Physiologic Data}

Extended cold storage followed by reperfusion produced consistent deleterious effects in lung performance among control animals. Specifically, arterial partial pressures of oxygen $\left(\mathrm{PaO}_{2}\right)$ and carbon dioxide $\left(\mathrm{PCO}_{2}\right)$ obtained from the left atrial cannulas consistently decreased over the 180 minutes of reperfusion (Table 1 and Figure 1). Control animals demonstrated increases in mean PAP during the study period as well (Table 1 and Figure 2).

Animals pretreated with oral tadalafil had higher $\mathrm{PaO}_{2}$ levels across the entire time of reperfusion when compared with control animals ( $P<.001$ by RM-ANOVA) (Table 1). In addition, post hoc testing revealed pretreatment to be associated with higher $\mathrm{PaO}_{2}$ levels at 90, 120, and 150 minutes after reperfusion by the Tukey honest significant difference test (Table 1). Random effects modeling suggested that tadalafil pretreatment was associated with an average $\mathrm{Po}_{2}$ increase of $117 \mathrm{~mm} \mathrm{Hg}(P=.02)$. By contrast, $\mathrm{PCO}_{2}$ levels were not different between groups (Figure 1, $B)$. Mean PAPs were lower in animals treated with tadalafil $(P=.006$ by RM-ANOVA $)$ and decreased by an average 17 $\mathrm{mm} \mathrm{Hg}(P=.008$ by GEE). Specific time points with decreased mean PAP included 120, 150, and 180 minutes after reperfusion (Figure 2). Wet/dry ratios did not differ between groups (10.3 in controls vs 10.0 PDE inhibition; $P=.86$ ).

\section{PDE Inhibition}

Control animals had increased PDE5 activity at both 1 and 3 hours after perfusion when compared with measurements taken before cold storage in the same animals $(P<.001$ for each). This translated into decreased levels of both cyclic GMP $(P<.01)$ and lower PKG activity $(P<.01)$ at 1 and 3 levels after perfusion when compared with prestorage levels. Animals preconditioned with oral tadalafil, retained PDE5A activity closer to baseline after 1 and 3 hours of perfusion. When PDE5A levels were examined between experimental groups, PDE5A activity at both 1 and 3 hours after perfusion was significantly greater in tadalafil-treated animals (Table 2). Consequently, cyclic GMP levels and PKG activity were significantly higher both before perfusion and at 1 and 3 hours after perfusion in tadalafil-treated animals when compared with controls.

\section{ROS}

Oral PDE inhibitor preconditioning produced significant decreases in levels of ROS (as measured by luminol chemiluminescence activity assays) at 1 and 3 hours after perfusion. Prestorage and preperfusion luminol activity was not different between groups (Figure 3).

\section{NOS Signaling}

Animals preconditioned with oral tadalafil demonstrated greater endothelial nitric oxide synthase (eNOS) activity 


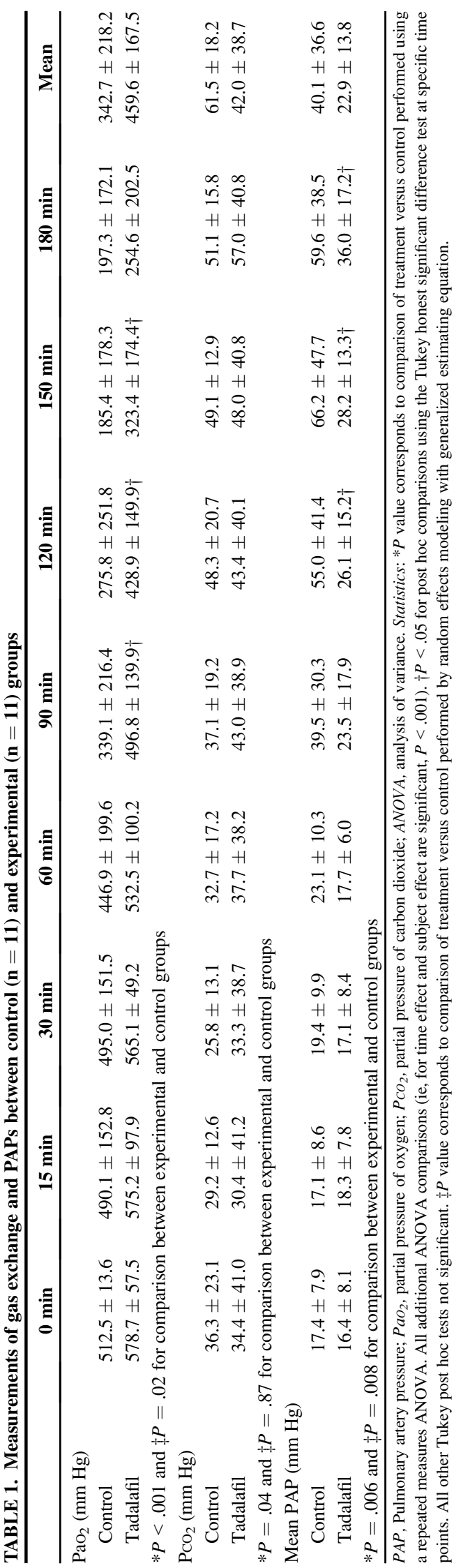

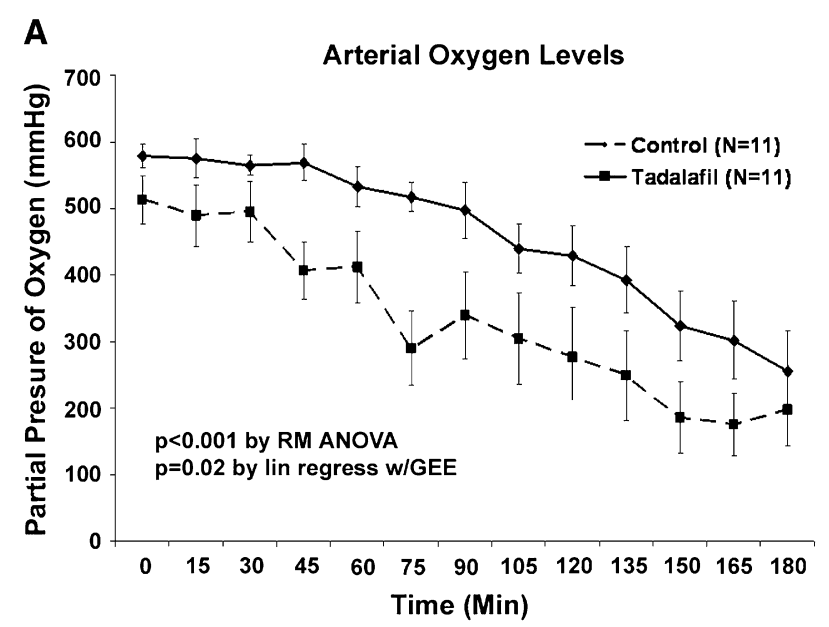

B

Arterial Carbon Dioxide Levels

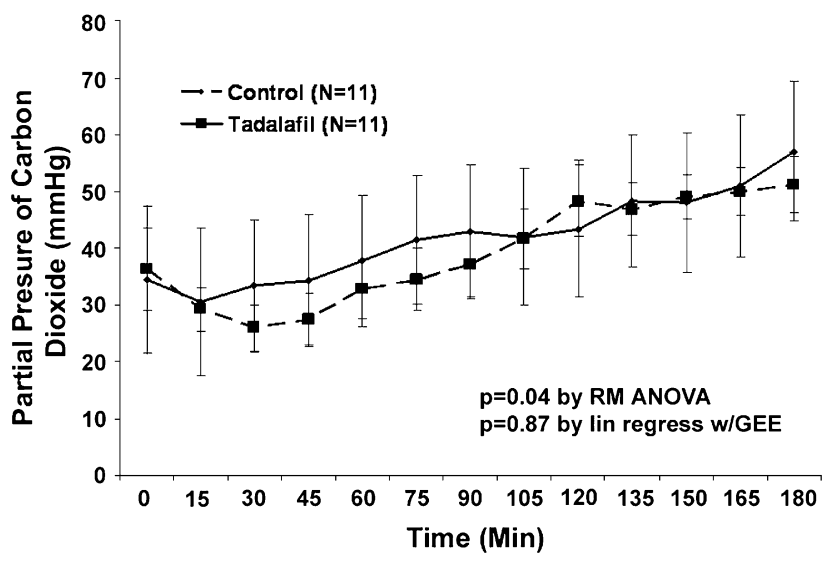

FIGURE 1. Partial pressures of arterial oxygen (A) and carbon dioxide (B) between control animals (dashed line) and animals treated with tadalafil (solid black line). Measurements by arterial blood gas sampling were taken at 15-minute intervals over the course of the experiment. Data points are mean values with error bars corresponding to standard error of the mean (SEM). $P$ values were obtained by repeated measures analysis of variance (RE ANOVA) between groups and modeling with generalized estimating equation $(G E E)$.

both before cold storage and at 1 and 3 hours after perfusion. Conversely, control animals exhibited greater increased inducible nitric oxide synthase (iNOS) activity at 1 and 3 hours after perfusion (Figure 4).

\section{DISCUSSION}

Our experimental model demonstrates that oral donor pretreatment with a long-acting PDE inhibitor improves early pulmonary performance after reperfusion. In our ex vivo design, rabbits preconditioned with oral tadalafil demonstrated improved oxygenation and manifested lower PAPs when compared with controls.

We have demonstrated that oral administration of tadalafil did in fact inhibit PDE5A activity in the lung. PDE5A 


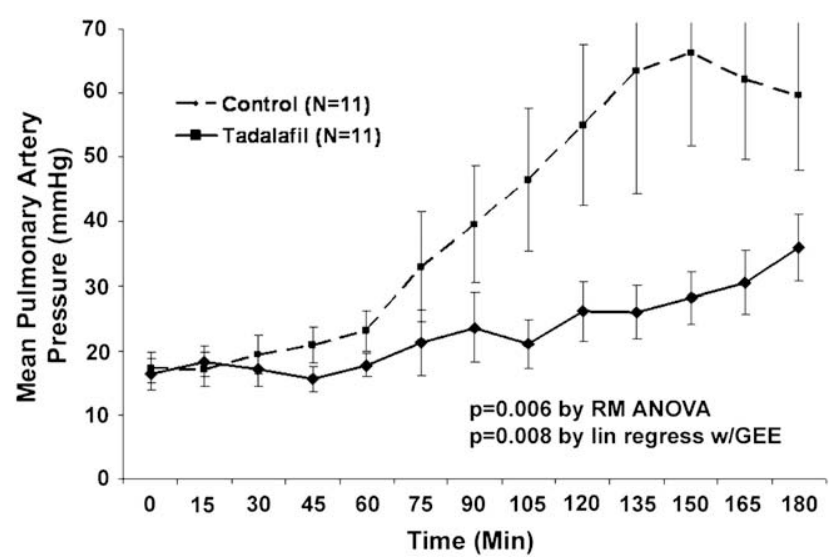

FIGURE 2. Mean pulmonary artery pressure (mPAP) between control animals (dashed line) and animals treated with tadalafil (solid black line). Measurements of mPAP were taken at 15-minute intervals over the course of the experiment. Data points are mean values with error bars corresponding to standard error of the mean (SEM). $P$ value corresponds to repeated measures analysis of variance ( $R M$ ANOVA) between groups modeling with generalized estimating equation $(G E E)$.

activity increased during reperfusion in control animals yet remained at baseline in our tadalafil-treated animals. As would be expected, increases in PDE5 activity in control animals translated to decreases in cyclic GMP and PKG. Additionally, animals preconditioned with tadalafil had increased levels of cyclic GMP and PKG.

Finally, we have shown that oxygen-derived free radicals (as measured by luminol chemiluminescence) are decreased with PDE inhibition. Oxygen-derived free radicals are important known mediators of IR injury of the lung. ${ }^{8}$ They can lead to direct cellular damage via activation of $\mathrm{Ca}^{2+}$ dependent proteases and also contribute to capillary leak and pulmonary edema. ${ }^{10,20}$ These findings taken together

TABLE 2. Markers of PDE inhibition in lung tissue

\begin{tabular}{lccc}
\hline & Control $(\mathbf{n}=\mathbf{9})$ & Tadalafil $(\mathbf{n}=\mathbf{6})$ & $\boldsymbol{P}$ value* \\
\hline PDE5A activity $(\mathrm{mP})$ & & & \\
$\quad$ Prestorage & $123.8 \pm 11.3$ & $124.8 \pm 11.4$ & .5 \\
Preperfusion & $137.5 \pm 5.3$ & $129.3 \pm 11.2$ & .3 \\
1 h after perfusion & $163 \pm 18.1$ & $134.8 \pm 7.2$ & .001 \\
3 $\mathrm{h}$ after perfusion & $181.2 \pm 31.7$ & $143.3 \pm 8.5$ & .001 \\
cGMP levels (pmol/mg) & & & \\
$\quad$ Prestorage & $1028.3 \pm 119.6$ & $1121.5 \pm 202.5$ & .87 \\
Preperfusion & $965.6 \pm 60.3$ & $1080 \pm 167.6$ & .04 \\
1 h after perfusion & $788.4 \pm 64.7$ & $1047 \pm 187.4$ & $<.001$ \\
3 h after perfusion & $809.5 \pm 123.2$ & $1137.2 \pm 475.3$ & .01 \\
PKG activity (fU/ $\mu \mathrm{g})$ & & & \\
Prestorage & $16.8 \pm 2.2$ & $20.2 \pm 3.2$ & .14 \\
Preperfusion & $16.4 \pm 1.2$ & $19.0 \pm 2.7$ & .03 \\
1 h after perfusion & $15.3 \pm 2.6$ & $19.0 \pm 7.1$ & $<.001$ \\
3 h after perfusion & $14.2 \pm 2.3$ & $25.4 \pm 12.2$ & .005 \\
\hline
\end{tabular}

PDE, Phosphodiesterase; $c G M P$, cyclic guanosine monophosphate $P K G$, protein kinase G; $f U$, fluorescent units. ${ }^{*} P$ value based on $t$ test comparisons for individual time points.

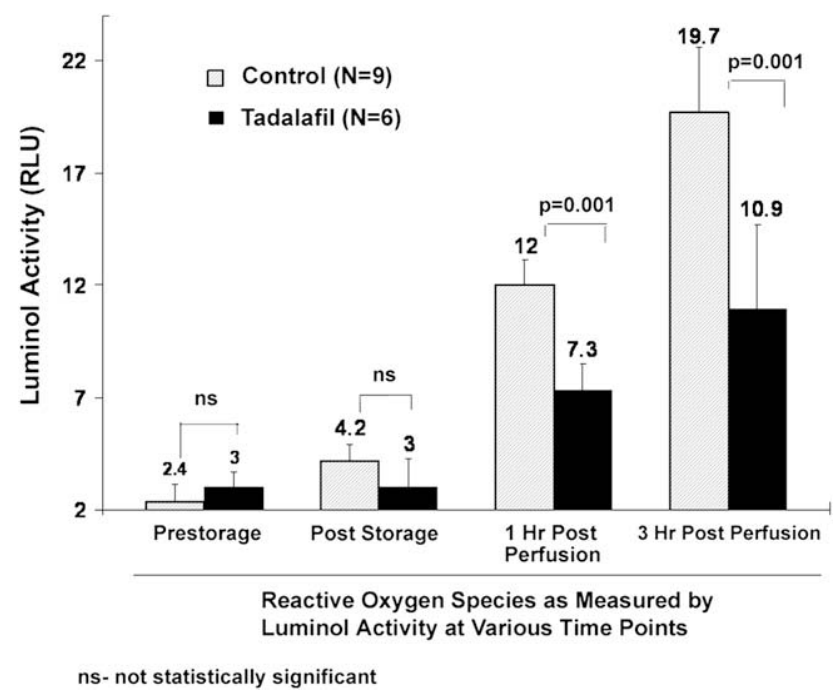

FIGURE 3. Levels of reactive oxygen species (ROS) as measured by luminal chemiluminescence activity (relative light units, $R L U$ ) at 4 distinct time points during the experiment. Control animals are seen in the gray shaded bars, and tadalafil-treated animals are shown with black solid bars. Means values are provided above the bars with error bars corresponding to standard deviations. $P$ values correspond to results obtained by comparison of means via the Student $t$ test.

suggest a role for donor PDE inhibition in the prevention and moderation of IR injury after lung transplantation.

The concept of using PDE inhibition against IR injury has been previously explored. Korom and colleagues ${ }^{21}$ treated donor pigs with intravenous sildenafil injected directly into the PA before experimental lung transplantation. In addition, sildenafil was placed in the perfusate solution. In their model, pigs that received sildenafil had improved shortterm survival. Pizanis and colleagues ${ }^{22}$ investigated the use of sildenafil administered both intravenously and in the flush solution before experimental transplantation. In this model, prerecovery PDE inhibition led to improved gas exchange in the transplanted organ. However, when sildenafil was also given in the preservation solution, it led to fatal graft failure. In another study by Dutly and associates, ${ }^{23}$ a selective PDE-4 enzyme inhibitor was introduced into flush solution before experimental lung transplantation. Treated animals demonstrated improved airway pressures, gas exchange, decreased edema, and minimal free radical injury.

Our study design is unique in that we used an oral preconditioning agent administered to donors hours before lung harvest. This strategy has advantages for clinical lung transplantation in humans, inasmuch as donor lung harvest is frequently delayed up to several hours owing to a variety of social, legal, administrative, and clinical factors. Our study is also unique in that we have made use of tadalafil as our PDE inhibitor. Because of its long half-life and excellent oral bioavailability, tadalafil is a drug ideally suited to donor 


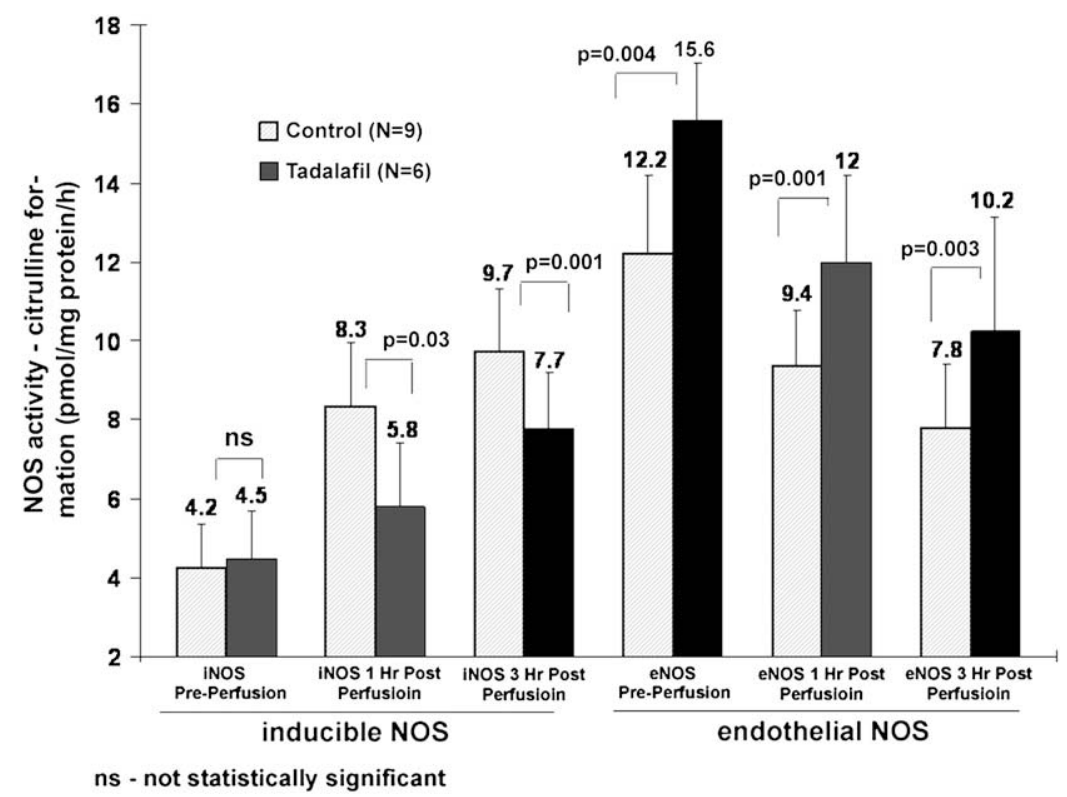

FIGURE 4. Nitric oxide synthase (NOS) activity for control (gray shaded bars) versus tadalafil-treated (solid black bars) animals as measured by citruline formation. Levels of both inducible NOS (iNOS) and endothelial NOS (eNOS) are provided. Means values are provided about the bars with error bars corresponding to standard deviations. $P$ values correspond to results obtained by comparison of means via the Student $t$ test.

pretreatment. We chose $1 \mathrm{mg}(0.25 \mathrm{mg} / \mathrm{kg})$ as our dose because it fell within the range of doses used in humans for erectile dysfunction (recommended dose $10-20 \mathrm{mg}, 0.15$ $0.29 \mathrm{mg} / \mathrm{kg}$ assuming 70-kg man, information from Lilly ICOS, www.cialis.com). Although this dose is slightly higher than the 0.5 - to $0.15-\mathrm{mg} / \mathrm{kg}$ range used in studies examining protection against IR injury with sildenafil, ${ }^{24}$ we believed that this higher dose was appropriate given oral administration with uncertain bioavailability. We chose to dose animals 6 hours before harvest and 24 hours before perfusion because tadalafil reaches peak concentrations at 120 minutes in a living organism and lasts up to 36 hours in the system.

\section{Mechanism of Protection With Selective PDE Inhibition}

Selective PDE5 inhibitors were developed for the treatment of erectile dysfunction in men. The principal mechanism of action involves modulation of NO-activated cyclic GMP by directly inhibiting the PDE enzyme responsible for cyclic GMP breakdown (Figure 5). The resulting increases in cyclic GMP leads to increased activation of PKG, which phosphorylates downstream targets including receptors, kinases, and phosphatases leading to a variety of cellular effects. ${ }^{12}$ PKG has been shown to alter vascular tone and can serve protective functions within the cell. PKG can in turn phosphorylate eNOS and iNOS, leading to feedback regulation of $\mathrm{NO}$ production. ${ }^{25,26}$

Although the biology of PDE activity in the lungs has not been firmly established, studies examining myocardial protection with PDE5A inhibitors have yielded important insights into potential mechanisms of protection. Specifically, preconditioning through active PDE inhibition with subsequent increases in cyclic GMP and PKG leads to a multitude of cellular protective effects (Figure 5). PKG can increase production of adenosine and bradykinin from endothelial cells, which may lead to donor pulmonary vasodilatation. This vasodilatation may translate into improved distribution of preservation solution at the time of recovery. PKG may also lead to opening of potassium channels $\left(\mathrm{K}_{\mathrm{ATP}}\right)$ on the inner mitochondrial membrane, which can contribute to cellular stabilization in the face of oxygen-derived free radical damage. ${ }^{24,27}$ Increases in cyclic GMP and eNOS may mediate platelet, neutrophil, macrophage, and leukocyte adhesion. Delayed protection may be a result of PDE5 activation of the $B c l-2$ gene, which has been shown to inhibit apoptosis. ${ }^{24}$

\section{NOS Signaling}

Our data suggest another important protective mechanism of PDE5 inhibition involving early preservation of eNOS activity and consequent decreases in ROS generation. In this experiment, both iNOS and eNOS activity were examined over several time points with treated animals demonstrating increases in eNOS activity during reperfusion. Conversely, reperfusion iNOS activity was significantly lower in tadalafil-treated animals. These data suggest a protective role of eNOS and injurious role of iNOS during lung reperfusion. Others have documented the importance of eNOS and iNOS in IR injury. Two studies from the University of 


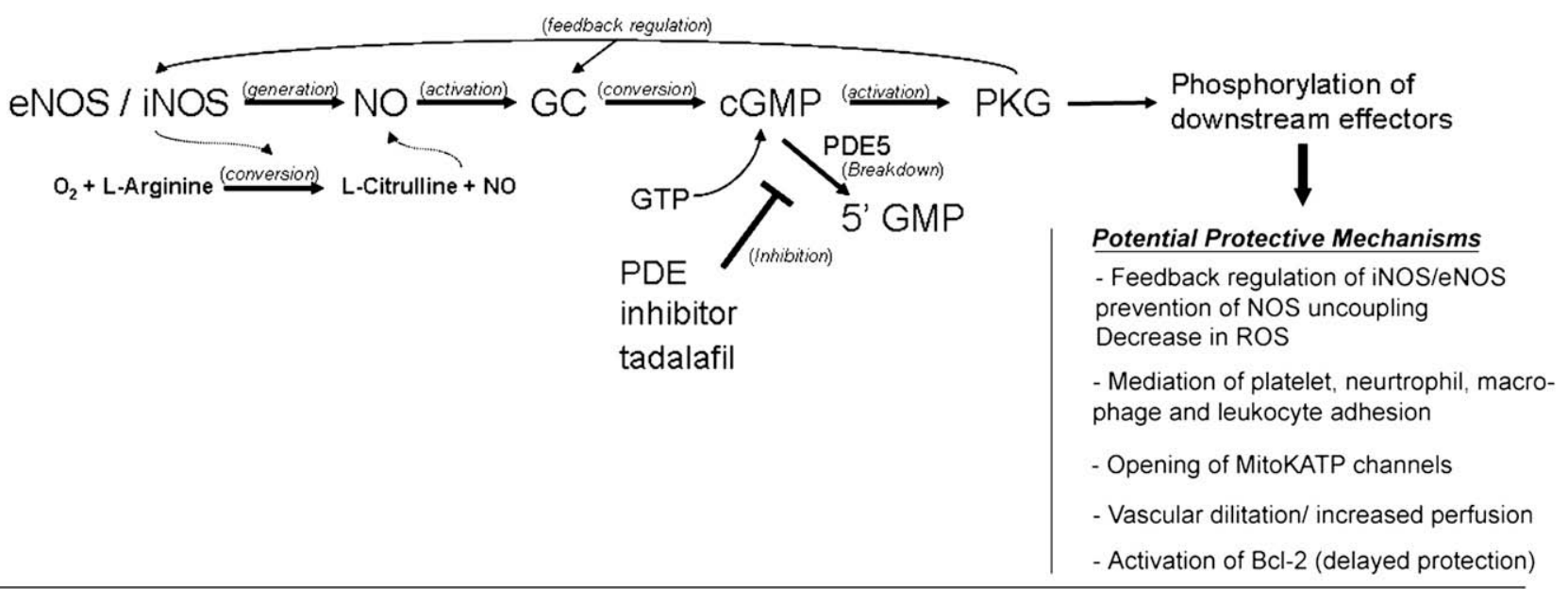

FIGURE 5. Mechanism of NOS signaling and PDE inhibition. Active inhibition of PDE5 by tadalafil leads to increases in cyclic GMP and PKG, which has a variety of potential protective effects. eNOS, Endothelial nitric oxide synthase; $i N O S$, inducible nitric oxide synthase; $\mathrm{O}_{2}$, oxygen; $\mathrm{NO}$, nitric oxide; $G C$, guanyl cyclase; $c G M P$, cyclic guanosine monophosphate; $G T P$, guanosine triphosphate; $P D E 5$, phosphodiesterase type 5 ; $P K G$, protein kinase G; ROS, reactive oxygen species; mitoKATP, mitochondrial potassium channels.

Toronto lung transplantation group have demonstrated that eNOS activity levels remain low during reperfusion, suggesting low eNOS levels may be an important mediator of injury after lung transplantation. ${ }^{28,29}$ In this group's experimental model, iNOS was elevated as well, suggesting a potentially injurious role. In additional studies, eNOS has been described as a critical regulator of NO signaling and important for the generation of ROS. This effect, known as NOS uncoupling, leads to ROS generation in multiple vascular beds. Donor PDE5 inhibition appears to maintain beneficial NOS coupling in our experimental model of lung reperfusion. This suggests that activation of PKG and its downstream targets may lead to stabilization of beneficial NO signaling and improved endothelial function.

\section{Role of PDE Biochemistry in Early Reperfusion Injury}

Reperfusion injury has been extensively studied, but our understanding of PDE biochemistry in lung transplantation continues to be limited. Pinksy and colleagues ${ }^{30}$ elegantly showed the benefits of cyclic GMP levels in early experimental allograft function using PDE5 inhibition. In this study, the investigators were unable to detect changes in NOS activity. Our study adds some new observations to the literature examining this issue. First, PDE5 activity specifically increases during early reperfusion with consequent decreases in cyclic GMP levels. This effect does not occur after cold storage alone. Furthermore, the increase in PDE5 activity correlates with a dramatic increase in oxidative stress (measured by ROS), decrease in gas exchange, and increase in PAPs. Increased PDE5 activity further corre- lates with decreased levels of cyclic GMP, and iNOS and eNOS undergo reciprocal changes in activity during early reperfusion, which is reversed by PDE5 inhibition.

From these observations, we can speculate that PDE5 activity is an important factor for early allograft dysfunction after lung transplantation. Furthermore, the increases in ROS that we have observed in this model suggest NOS uncoupling occurs early during reperfusion. Thus, PDE5, by decreasing levels of cyclic GMP and PKG, likely plays a crucial role in disturbing the balance between eNOS and iNOS activity after lung transplantation. These two enzymes may be pivotal for influencing the magnitude of pulmonary injury occurring from reperfusion and PDE5 inhibitors may help to restore normal balance and prevent IR injury.

\section{Study Limitations}

Our study is limited by use of a model that is ex vivo in nature. We do not use immunosuppression, and our animals have not undergone brain death before harvest. Thus, our model does not completely replicate in vivo lung transplantation. Inasmuch as we reperfuse for only 3 hours, the effects we have observed with PDE inhibition may be short-lived, and we do not know the effects of this therapy for longterm survival. Further, we do not know whether we have conducted our experiments using the optimal dose of our PDE inhibitor. Although we observed a drug effect, we based our dosing on human dosing and literature, and we do not know whether a more optimal dose could have been used or whether dosing should be repeated. We therefore will focus future experimentation on dosing levels as well as redosing. Despite these limitations, we have 
achieved a reliable model capable of producing severe reperfusion injury of the lungs similar to that seen in clinical lung transplantation.

\section{CONCLUSIONS}

IR injury remains a substantial cause of morbidity and mortality after lung transplantation. Novel methods to protect allografts during recovery will not only decrease the incidence of PGD and chronic graft failure, but also may embolden centers to use marginal donors with increased ischemic times, thus increasing the currently limited donor supply. In this study, we have examined the use of a longacting oral PDE inhibitor for donor preconditioning in a model of IR injury. Our data demonstrate that this strategy is effective for improving pulmonary performance and decreasing oxygen-derived free radical levels after reperfusion. PDE enzymes and their downstream effectors may play a critical role in reperfusion injury after lung transplantation.

We thank Mr Jeffrey Brawn, Mrs Melissa Jones, and Ms Tamara Treat for their outstanding technical assistance. We acknowledge Dr Irving L. Kron and associates at the University of Virginia for providing guidance and expertise in setting up the ex vivo perfusion apparatus at our institution.

\section{References}

1. Christie JD, Bavaria JE, Palevsky HI, Litzky L, Blumenthal NP, Kaiser LR, et al. Primary graft failure following lung transplantation. Chest. 1998;114:51-60.

2. King RC, Binns OA, Rodriguez F, Kanithanon RC, Daniel TM, Spotnitz WD, et al. Reperfusion injury significantly impacts clinical outcome after pulmonary transplantation. Ann Thorac Surg. 2000;69:1681-5.

3. Granton J. Update of early respiratory failure in the lung transplant recipient. Curr Opin Crit Care. 2006;12:19-24.

4. Whitson BA, Prekker ME, Herrington CS, Whelan TP, Radosevich DM, Hertz MI, et al. Primary graft dysfunction and long-term pulmonary function after lung transplantation. J Heart Lung Transplant. 2007;26:1004-11.

5. Whitson BA, Nath DS, Johnson AC, Walker AR, Prekker ME, Radosevich DM, et al. Risk factors for primary graft dysfunction after lung transplantation. $J$ Thorac Cardiovasc Surg. 2006;131:73-80.

6. de Perrot M, Liu M, Waddell TK, Keshavjee S. Ischemia-reperfusion-induced lung injury. Am J Respir Crit Care Med. 2003;167:490-511.

7. Daud SA, Yusen RD, Meyers BF, Chakinala MM, Walter MJ, Aloush AA, et al. Impact of immediate primary lung allograft dysfunction on bronchiolitis obliterans syndrome. Am J Respir Crit Care Med. 2007;175:507-13.

8. Ng CS, Wan S, Arifi AA, Yim AP. Inflammatory response to pulmonary ischemia-reperfusion injury. Surg Today. 2006;36:205-14.

9. Vural KM, Oz MC. Endothelial adhesivity, pulmonary hemodynamics and nitric oxide synthesis in ischemia-reperfusion. Eur J Cardiothorac Surg. 2000;18: 348-52.

10. Schutte H, Witzenrath M, Mayer K, Weissmann N, Schell A, Rosseau S, et al. The PDE inhibitor zaprinast enhances NO-mediated protection against vascular leakage in reperfused lungs. Am J Physiol. 2000;279:L496-502.

11. Featherstone RL, Chambers DJ, Kelly FJ. Comparison of phosphodiesterase inhibitors of differing isoenzyme selectivity added to St. Thomas' hospital cardioplegic solution used for hypothermic preservation of rat lungs. Am J Respir Crit Care Med. 2000;162(3 Pt 1):850-6.

12. Kass DA, Takimoto E, Nagayama T, Champion HC. Phosphodiesterase regulation of nitric oxide signaling. Cardiovasc Res. 2007;75:303-14.

13. Masson P, Lambert SM, Brown M, Shabsigh R. PDE-5 inhibitors: current status and future trends. Urol Clin North Am. 2005;32:511-25, viii.

14. Porst H. IC351 (tadalafil, Cialis): update on clinical experience. Int J Impot Res. 2002;14(Suppl. 1):S57-64.
15. Shabsigh R, Seftel AD, Rosen RC, Porst H, Ahuja S, Deeley MC, et al. Review of time of onset and duration of clinical efficacy of phosphodiesterase type 5 inhibitors in treatment of erectile dysfunction. Urology. 2006;68:689-96.

16. Hemnes AR, Zaiman A, Champion HC. PDE5A inhibition attenuates bleomycininduced pulmonary fibrosis and pulmonary hypertension through inhibition of ROS generation and RhoA/Rho kinase activation. Am J Physiol. 2008;294: L24-33.

17. Bivalacqua TJ, Burnett AL, Hellstrom WJ, Champion HC. Overexpression of arginase in the aged mouse penis impairs erectile function and decreases eNOS activity: influence of in vivo gene therapy of anti-arginase. Am J Physiol Heart Circ Physiol. 2007;292:H1340-51.

18. Tankersley CG, Champion HC, Takimoto E, Gabrielson KL, Bedja D, Misra V, et al. Exposure to inhaled particulate matter impairs cardiac function in senescent mice. Am J Physiol Regul Integr Comp Physiol. 2008;295:R252-63.

19. Hsu LL, Champion HC, Campbell-Lee SA, Bivalacqua TJ, Manci EA, Diwan BA, et al. Hemolysis in sickle cell mice causes pulmonary hypertension due to global impairment in nitric oxide bioavailability. Blood. 2007;109: 3088-98.

20. Eckenhoff RG, Dodia C, Tan Z, Fisher AB. Oxygen-dependent reperfusion injury in the isolated rat lung. J Appl Physiol. 1992;72:1454-60.

21. Korom S, Hillinger S, Cardell M, Zhai W, Tan Q, Dutly A, et al. Sildenafil extends survival and graft function in a large animal lung transplantation model. Eur $J$ Cardiothorac Surg. 2006;29:288-93.

22. Pizanis N, Milekhin V, Tsagakis K, Aleksic I, Kamler M, Jakob H. PDE-5 inhibitor donor intravenous preconditioning is superior to supplementation in standard preservation solution in experimental lung transplantation. Eur J Cardiothorac Surg. 2007;32:42-7.

23. Dutly AE, Inci I, Hillinger S, Zalunardo M, Gaspert A, Rousson V, et al. Normal gas exchange after 30 -h ischemia and treatment with phosphodiesterase inhibitor PDI747. Eur J Cardiothorac Surg. 2003;24:594-600.

24. Kukreja RC, Salloum F, Das A, Ockaili R, Yin C, Bremer YA, et al. Pharmacological preconditioning with sildenafil: basic mechanisms and clinical implications. Vasc Pharmacol. 2005;42:219-32.

25. Kukreja RC, Xi L. eNOS phosphorylation: a pivotal molecular switch in vasodilation and cardioprotection? J Mol Cell Cardiol. 2007;42:280-2.

26. Mount PF, Kemp BE, Power DA. Regulation of endothelial and myocardial NO synthesis by multi-site eNOS phosphorylation. J Mol Cell Cardiol. 2007;42: 271-9.

27. Kukreja RC. Cardiovascular protection with sildenafil following chronic inhibition of nitric oxide synthase. Br J Pharmacol. 2007;150:538-40.

28. Cardella JA, Keshavjee SH, Bai XH, Yeoh JS, Granton JT, Meade MO, et al. Increased expression of nitric oxide synthase in human lung transplants after nitric oxide inhalation. Transplantation. 2004;77:886-90.

29. Liu M, Tremblay L, Cassivi SD, Bai XH, Mourgeon E, Pierre AF, et al. Alterations of nitric oxide synthase expression and activity during rat lung transplantation. Am J Physiol. 2000;278:L1071-81.

30. Pinsky DJ, Naka Y, Chowdhury NC, Liao H, Oz MC, Michler RE, et al. The nitric oxide/cyclic GMP pathway in organ transplantation: critical role in successful lung preservation. Proc Natl Acad Sci U S A. 1994;91:12086-90.

\section{Discussion}

Dr Christine L. Lau (Charlottesville, $\mathrm{Va}$ ). My congratulations to Dr Weiss and the Johns Hopkins group for another outstanding presentation in the area of lung transplantation.

IR injury remains a major morbidity and potential mortality early after lung transplantation, and, importantly, now it is known to be a major risk factor for the development of bronchiolitis obliterans. In this current study, the authors show in an experimental ex vivo lung transplant model that oral preconditioning of the donor with a PDE inhibitor leads to a decrease in IR injury, as evidenced by markers of $\mathrm{PaO}_{2}$, PAP, and ROS. As pointed out, however, the concept of PDE inhibitors to limit IR injury is not entirely new. Others have shown similar benefits in the treatment of IR to those described in this study with the use of intravenous sildenafil as well as with other PDE inhibitors, specifically PDE-4 inhibitors. This current study, however, does add to the previous investigations 
by looking at an oral long-acting agent and thus has some obvious advantages.

I have two questions. As you know, the primary effect of PDE-5 activity is on cyclic GMP levels and, in turn, stimulation of PKG. Cyclic GMP also can influence other PDEs that in turn regulate cyclic adenosine monophosphate and, thus, lung function. Do you plan to evaluate the synergy with other cyclic nucleotide PDEs, for example, PDE-4 inhibition? Second, what are your plans for future studies? Do you plan to look at leukocyte trafficking and platelet function, histology, and immunohistochemistry, and do you plan any clinical trials?

Dr Weiss. Thank you, Dr Lau, for your critique and for your questions and comments.

You bring up an excellent point regarding PDE-4. Certainly others, including excellent work from the investigators at the University of Virginia, have looked at activation of the PDE-4 enzyme in models of IR injury. To contrast the difference between PDE-4 and PDE-5, PDE-4 breaks down cyclic adenosine and PDE-5 breaks down cyclic GMP; PDE-4 is generally found in inflammatory cells whereas PDE-5 is generally located in endothelial cells. Although these are generalizations, thinking about the enzymes in that manner can help us understand their principal activity. We do not know to what extent tadalafil acts on PDE-4. I suspect that there is some synergy, and we do plan to investigate that aspect in future experiments. The answer to your question is yes. I think that this brings up an opportunity for dual therapy with both PDE-5 and PDE-4 inhibitors. We would be interested in exploring that.
In terms of our future directions, we want to make our model more clinically applicable. For example, we are interested in examining brain death in our model and we are interested in examining the effect in a long-term survival model. There is some indication from our data that the effect may be short-lived, and so we need to focus on dosing and redosing as well. We are also interested in looking at platelet function. Finally, we want to examine the role of PDE enzymes in human lung tissue. We have an approved protocol at our institution to examine human lung tissue after reperfusion that would have otherwise been discarded. These are some of the future directions that we are interested in exploring.

Dr Yolonda L. Colson (Boston, Mass). Is most of this effect just owing to changes in platelet function with your perfusion with the drug on board, and do you have a control to control for changes in platelet function?

Dr Weiss. Thank you, Dr Colson, for that question. Although we know that PDE inhibition can affect platelet function, it is not likely in our model inasmuch as we are pretreating the donor. We flush out most of the blood during recovery and it is therefore unlikely that recipient platelets are exposed to the drug.

Dr. Colson. You could argue that the PDE is just changing platelet function, and if you are not having microemboli, your PAP is lower and your oxygenation is higher.

Dr Weiss. That is true. We did not measure recipient serum levels of PDE inhibition to prove that the platelets are unaffected. I think your point is well taken and may be a good reason to give PDE inhibitors to recipients before transplantation, to decrease injurious platelet behavior. 